\title{
FACTORING DERIVATIVES OF FUNCTIONS IN THE NEVANLINNA AND SMIRNOV CLASSES
}

\author{
Konstantin M. Dyakonov
}

\author{
ICREA and Universitat de Barcelona, Departament de Matemàtica Aplicada i Anàlisi \\ Gran Via 585, E-08007 Barcelona, Spain; konstantin.dyakonov@icrea.cat
}

\begin{abstract}
We prove that, given a function $f$ in the Nevanlinna class $\mathcal{N}$ and a positive integer $n$, there exist $g \in \mathcal{N}$ and $h \in$ BMOA such that $f^{(n)}=g h^{(n)}$. We may choose $g$ to be zero-free, so it follows that the zero sets for the class $\mathcal{N}^{(n)}:=\left\{f^{(n)}: f \in \mathcal{N}\right\}$ are the same as those for BMOA ${ }^{(n)}$. Furthermore, while the set of all products $g h^{(n)}$ (with $g$ and $h$ as above) is strictly larger than $\mathcal{N}^{(n)}$, we show that the gap is not too large, at least when $n=1$. Precisely speaking, the class $\left\{g h^{\prime}: g \in \mathcal{N}, h \in \mathrm{BMOA}\right\}$ turns out to be the smallest ideal space containing $\left\{f^{\prime}: f \in \mathcal{N}\right\}$, where "ideal" means invariant under multiplication by $H^{\infty}$ functions. Similar results are established for the Smirnov class $\mathcal{N}^{+}$.
\end{abstract}

\section{Introduction and results}

Let $\mathcal{H}(\mathbf{D})$ stand for the set of holomorphic functions on the disk $\mathbf{D}:=\{z \in \mathbf{C}$ : $|z|<1\}$. Given a class $X \subset \mathcal{H}(\mathbf{D})$ and an integer $n \in \mathbf{N}:=\{1,2, \ldots\}$, we write

$$
X^{(n)}:=\left\{f^{(n)}: f \in X\right\},
$$

where $f^{(n)}$ is the $n$th derivative of $f$. When $n=1$, we also use the notation $X^{\prime}$ instead of $X^{(1)}$. Further, we denote by $\mathcal{Z}(X)$ the collection of zero sets for $X$; a (discrete) subset $E$ of $\mathbf{D}$ will thus belong to $\mathcal{Z}(X)$ if and only if $E=\{z \in \mathbf{D}: f(z)=0\}$ for some non-null function $f \in X$. Now, if $X$ and $Y$ are subclasses of $\mathcal{H}(\mathbf{D})$, we put

$$
X \cdot Y:=\{f g: f \in X, g \in Y\} .
$$

Finally, a vector space $X$ contained in $\mathcal{H}(\mathbf{D})$ is said to be ideal if

$$
H^{\infty} \cdot X \subset X,
$$

where, as usual, $H^{\infty}$ is the space of bounded holomorphic functions on D.

Our starting point is a result of Cohn and Verbitsky [3] which asserts, or rather implies, that

$$
\left(H^{p}\right)^{(n)}=H^{p} \cdot \mathrm{BMOA}^{(n)}
$$

whenever $0<p<\infty$ and $n \in \mathbf{N}$. Here, we write $H^{p}$ for the classical (holomorphic) Hardy spaces on the disk, and BMOA for the "analytic subspace" of BMO $=\mathrm{BMO}(\mathbf{T})$, the space of functions with bounded mean oscillation on the circle $\mathbf{T}:=\partial \mathbf{D}$. Precisely speaking, BMOA can be defined as $H^{1} \cap \mathrm{BMO}$; as to the definitions of (and background information on) $H^{p}$ and $\mathrm{BMO}$, the reader will find these standard matters in [5, Chapters II and VI].

doi:10.5186/aasfm.2012.3728

2010 Mathematics Subject Classification: Primary 30D50, 30D55.

Key words: Nevanlinna class, Smirnov class, BMOA, derivatives, factorization, zero sets.

Supported in part by grant MTM2011-27932-C02-01 from El Ministerio de Ciencia e Innovación (Spain) and grant 2009-SGR-1303 from AGAUR (Generalitat de Catalunya). 
For $n=1$, identity (1.1) was first obtained in Cohn's earlier paper [2]. It was then extended in [3] to higher order (possibly fractional) derivatives and still further; indeed, more general factorization theorems involving tent spaces - and Triebel spaces - were actually established there. It was also shown in [3] that, when factoring $f^{(n)}$ for $f \in H^{p}$ in the sense of (1.1), one may choose the $H^{p}$ factor on the right to be an outer function. As a consequence, one sees that

$$
\mathcal{Z}\left(\left(H^{p}\right)^{(n)}\right)=\mathcal{Z}\left(\operatorname{BMOA}^{(n)}\right)
$$

In particular, for any fixed $n$, the zero sets for $\left(H^{p}\right)^{(n)}$ are the same for all $p \in$ $(0, \infty)$. This last fact was contrasted in [3] with the Bergman space situation, where different $A^{p}$ spaces happen to have different zero sets; see [7]. We wish to add, in this connection, that a similar Bergman-type phenomenon (different zero sets for different $p$ 's) is also encountered in certain "small" $H^{p}$-related spaces; namely, it occurs [4] for the star-invariant subspaces $H^{p} \cap \theta \overline{H_{0}^{p}}$ associated with an inner function $\theta$.

Also related to (1.1), in the case $n=1$, is Aleksandrov and Peller's work from [1]. There, for a given $f \in H^{p}$, a weak factorization $f^{\prime}=\sum_{j=1}^{m} g_{j} h_{j}^{\prime}$ was constructed with suitable $g_{j} \in H^{p}$ and $h_{j} \in H^{\infty}$. This was done with $m=2$ for $1<p<\infty$, with $m=4$ for $p=1$, and with a certain larger $m$ for $0<p<1$. Yet another weak factorization theorem from [1], which establishes a connection between BMOA' and $\left(H^{\infty}\right)^{\prime}$, will be employed in Section 4 below.

The purpose of this paper is to find out whether - and/or to which extent - the (strong) factorization theorem (1.1) carries over to the Nevanlinna class $\mathcal{N}$, or the Smirnov class $\mathcal{N}^{+}$, in place of $H^{p}$.

Let us recall that $\mathcal{N}$ is defined as the set of functions $f \in \mathcal{H}(\mathbf{D})$ with

$$
\sup _{0<r<1} \int_{\mathbf{T}} \log ^{+}|f(r \zeta)||d \zeta|<\infty
$$

while $\mathcal{N}^{+}$is formed by those $f \in \mathcal{N}$ which satisfy

$$
\lim _{r \rightarrow 1^{-}} \int_{\mathbf{T}} \log ^{+}|f(r \zeta)||d \zeta|=\int_{\mathbf{T}} \log ^{+}|f(\zeta)||d \zeta| .
$$

Equivalently, the elements of $\mathcal{N}$ (resp., $\mathcal{N}^{+}$) are precisely the ratios $u / v$, with $u, v \in$ $H^{\infty}$ and with $v$ nonvanishing (resp., outer) on $\mathbf{D}$; for this and other characterizations of the two classes, see [5, Chapter II].

As far as factorization theorems of the form (1.1) are concerned, we can hardly expect the behavior of $\mathcal{N}$ or $\mathcal{N}^{+}$to mimic that of $H^{p}$ too closely. In fact, as we shall soon explain, it is the "easy" part of (1.1), i.e., the inclusion

$$
\left(H^{p}\right)^{(n)} \supset H^{p} \cdot \mathrm{BMOA}^{(n)}
$$

that admits no extension to the Nevanlinna or Smirnov setting. Meanwhile, we remark that (1.3) is indeed easy to deduce, at least for $p=2$, from the (not so easy, but readily available) descriptions of $\left(H^{p}\right)^{(n)}$ and $\mathrm{BMOA}^{(n)}$ as the appropriate Triebel spaces; see [11]. One of these tells us that, for $\varphi \in \mathcal{H}(\mathbf{D})$,

$$
\varphi \in\left(H^{p}\right)^{(n)} \Longleftrightarrow \int_{\mathbf{T}}\left(\int_{0}^{1}|\varphi(r \zeta)|^{2}(1-r)^{2 n-1} d r\right)^{p / 2}|d \zeta|<\infty
$$


for all $n \in \mathbf{N}$ and $0<p<\infty$, a fact that has no counterpart for $\mathcal{N}$ or $\mathcal{N}^{+}$. The other, which involves a Carleson measure characterization of BMOA, will be mentioned in Section 2 below.

Now, to see that the $\mathcal{N}$ and $\mathcal{N}^{+}$versions of (1.3) actually break down, already for $n=1$, one may recall results of Hayman [6] and Yanagihara [12] saying that neither $\mathcal{N}$ nor $\mathcal{N}^{+}$is invariant with respect to integration. More precisely, Hayman gave an example of a function $f \in \mathcal{N}$ whose antiderivative $F(z):=\int_{0}^{z} f(\zeta) d \zeta$ is not in $\mathcal{N}$, and Yanagihara refined this by showing that $F$ need not be in $\mathcal{N}$ even for $f \in \mathcal{N}^{+}$. Consequently, $\mathcal{N}^{+}$is not contained in $\mathcal{N}^{\prime}$, whence a fortiori

$$
\mathcal{N} \not \subset \mathcal{N}^{\prime} \text { and } \mathcal{N}^{+} \not \subset\left(\mathcal{N}^{+}\right)^{\prime} \text {. }
$$

Since $\mathcal{N} \cdot \mathrm{BMOA}^{\prime}\left(\right.$ resp., $\mathcal{N}^{+} \cdot \mathrm{BMOA}^{\prime}$ ) contains $\mathcal{N}\left(\right.$ resp., $\mathcal{N}^{+}$), we readily deduce from (1.4) that

$$
\mathcal{N} \cdot \mathrm{BMOA}^{\prime} \not \subset \mathcal{N}^{\prime} \text { and } \mathcal{N}^{+} \cdot \mathrm{BMOA}^{\prime} \not \subset\left(\mathcal{N}^{+}\right)^{\prime} .
$$

A similar conclusion holds for higher order derivatives as well.

We prove, however, that the "difficult" part of (1.1), i.e., the inclusion

$$
\left(H^{p}\right)^{(n)} \subset H^{p} \cdot \mathrm{BMOA}^{(n)}
$$

does remain valid with either $\mathcal{N}$ or $\mathcal{N}^{+}$in place of $H^{p}$.

Theorem 1.1. For each $n \in \mathbf{N}$, we have

$$
\mathcal{N}^{(n)} \subset \mathcal{N} \cdot \mathrm{BMOA}^{(n)} \text { and }\left(\mathcal{N}^{+}\right)^{(n)} \subset \mathcal{N}^{+} \cdot \mathrm{BMOA}^{(n)} .
$$

Moreover, given $f \in \mathcal{N}$ (resp., $f \in \mathcal{N}^{+}$), one can find a zero-free function $g \in \mathcal{N}$ (resp., an outer function $g \in \mathcal{N}^{+}$) and an $h \in$ BMOA such that $f^{(n)}=g h^{(n)}$.

It should be mentioned that our method also applies to the meromorphic Nevanlinna class $\mathcal{N}_{\text {mer }}$, defined as the set of quotients $u / v$, where $u, v \in H^{\infty}$ and $v$ is merely required to be non-null. In fact, a glance at our proof of Theorem 1.1 will reveal that if the original function $f$ is of the form $F / I$, with $F \in \mathcal{N}^{+}$and $I$ inner, then we may take $g=G / I^{n+1}$, with $G$ outer. And again, just as in the $H^{p}$ setting, our factorization theorem yields information on the zero sets.

Corollary 1.2. We have

$$
\mathcal{Z}\left(\mathcal{N}^{(n)}\right)=\mathcal{Z}\left(\mathrm{BMOA}^{(n)}\right), \quad n \in \mathbf{N} .
$$

Indeed, Theorem 1.1 shows that every zero set for $\mathcal{N}^{(n)}$ is a zero set for $\mathrm{BMOA}^{(n)}$, while the converse is immediate from the fact that BMOA $\subset \mathcal{N}$. Furthermore, since $\mathcal{N}^{+}$lies between BMOA and $\mathcal{N}$, as does every $H^{p}$ with $0<p<\infty$, Corollary 1.2 obviously implies the identity

$$
\mathcal{Z}\left(\left(\mathcal{N}^{+}\right)^{(n)}\right)=\mathcal{Z}\left(\operatorname{BMOA}^{(n)}\right)
$$

and also (1.2).

Finally, restricting ourselves to the case $n=1$, we wish to take a closer look at the inclusion

$$
\mathcal{N}^{\prime} \subset \mathcal{N} \cdot \mathrm{BMOA}^{\prime}
$$

from Theorem 1.1, along with its $\mathcal{N}^{+}$counterpart. We know from (1.5) that the inclusion is proper, and we now stress an important point of distinction between the two sides. Namely, the right-hand side, $\mathcal{N} \cdot \mathrm{BMOA}^{\prime}$, is ideal (i.e., invariant under 
multiplication by $H^{\infty}$ functions), whereas the left-hand side, $\mathcal{N}^{\prime}$, is not. Moreover, the space $\mathcal{N}^{\prime}$ is highly nonideal in the sense that even the identity function $z$ is not a multiplier thereof! (Otherwise, the formula

$$
g=(z g)^{\prime}-z g^{\prime}, \quad g \in \mathcal{N},
$$

would imply that $\mathcal{N}$ is contained in $\mathcal{N}^{\prime}$, which we know is false.) A similar remark applies to $\left(\mathcal{N}^{+}\right)^{\prime}$.

Our last result states, then, that $\mathcal{N} \cdot \mathrm{BMOA}^{\prime}$ is actually the smallest ideal space containing $\mathcal{N}^{\prime}$, and that the same is true in the $\mathcal{N}^{+}$setting.

Theorem 1.3. (a) The class $\mathcal{N} \cdot \mathrm{BMOA}^{\prime}$ is the ideal hull of $\mathcal{N}^{\prime}$. In other words, $\mathcal{N} \cdot$ BMOA $^{\prime}$ is an ideal vector space that contains $\mathcal{N}^{\prime}$ and is contained in every ideal space $X$ with $\mathcal{N}^{\prime} \subset X$.

(b) Similarly, $\mathcal{N}^{+} \cdot \mathrm{BMOA}^{\prime}$ is the ideal hull of $\left(\mathcal{N}^{+}\right)^{\prime}$.

Now let us turn to the proofs.

\section{Preliminaries}

A couple of lemmas will be needed.

Lemma 2.1. Let $k \geq 0$ and $l \geq 1$ be integers. If $\varphi \in \mathrm{BMOA}^{(l)}$ and $\psi$ is a function in $\mathcal{H}(\mathbf{D})$ satisfying

$$
\psi(z)=O\left((1-|z|)^{-k}\right), \quad z \in \mathbf{D},
$$

then $\varphi \psi \in \mathrm{BMOA}^{(k+l)}$.

Proof. It is known (see, e.g., $[8,10,11]$ ) that a function $F \in \mathcal{H}(\mathbf{D})$ will be in $\mathrm{BMOA}^{(n)}$, with $n \in \mathbf{N}$, if and only if the measure $|F(z)|^{2}(1-|z|)^{2 n-1} d x d y$ (where $z=x+i y)$ is a Carleson measure. The required result follows from this immediately, since (2.1) yields

$$
|\varphi(z) \psi(z)|^{2}(1-|z|)^{2(k+l)-1} \leq \mathrm{const} \cdot|\varphi(z)|^{2}(1-|z|)^{2 l-1}
$$

for all $z \in \mathbf{D}$.

When $k=0$, the above lemma reduces to saying that

$$
H^{\infty} \cdot \operatorname{BMOA}^{(n)} \subset \mathrm{BMOA}^{(n)}
$$

for all $n \in \mathbf{N}$; in other words, $\mathrm{BMOA}^{(n)}$ is an ideal space. This in turn leads to the next observation.

Lemma 2.2. For each $n \in \mathbf{N}$, the sets $\mathcal{N} \cdot \mathrm{BMOA}^{(n)}$ and $\mathcal{N}^{+} \cdot \mathrm{BMOA}^{(n)}$ are ideal vector spaces.

Proof. It is clear that the two sets are invariant under multiplication by $H^{\infty}$ functions, but maybe not quite obvious that they are vector spaces. It is the linearity property

$$
f_{1}, f_{2} \in \mathcal{N} \cdot \operatorname{BMOA}^{(n)} \Longrightarrow f_{1}+f_{2} \in \mathcal{N} \cdot \operatorname{BMOA}^{(n)}
$$

(and a similar fact with $\mathcal{N}^{+}$in place of $\mathcal{N}$ ) that should be verified. To this end, we write

$$
f_{j}=\frac{u_{j}}{v_{j}} \cdot w_{j}^{(n)} \quad(j=1,2),
$$


where $u_{j}, v_{j} \in H^{\infty}$ and $w_{j} \in \mathrm{BMOA}$, and where $v_{j}$ is zero-free (resp., outer if the $f_{j}$ 's are from $\left.\mathcal{N}^{+} \cdot \mathrm{BMOA}^{(n)}\right)$. Note that

$$
f_{1}+f_{2}=\frac{1}{v_{1} v_{2}} \cdot\left(u_{1} v_{2} w_{1}^{(n)}+u_{2} v_{1} w_{2}^{(n)}\right) \text {. }
$$

The two terms in brackets, and hence their sum, will be in $\mathrm{BMOA}^{(n)}$ by virtue of (2.2), while the factor $1 /\left(v_{1} v_{2}\right)$ will be in $\mathcal{N}$ (resp., in $\left.\mathcal{N}^{+}\right)$.

\section{Proof of Theorem 1.1}

We treat the case of $\mathcal{N}$ first. Take $f \in \mathcal{N}$ and write $f=u / v$, where $u, v \in H^{\infty}$ and $v$ has no zeros in $\mathbf{D}$. We have then

$$
f^{(n)}=\sum_{k=0}^{n}\left(\begin{array}{l}
n \\
k
\end{array}\right) u^{(n-k)}(1 / v)^{(k)} .
$$

For each $k \in\{0, \ldots, n\}$, Faà di Bruno's formula (see [9, Chapter 3]) yields

$$
\left(\frac{1}{v}\right)^{(k)}=\sum C\left(m_{1}, \ldots, m_{k}\right) v^{-m_{1}-\cdots-m_{k}-1} \prod_{j=1}^{k}\left(v^{(j)}\right)^{m_{j}},
$$

where the sum is over the $k$-tuples $\left(m_{1}, \ldots, m_{k}\right)$ of nonnegative integers satisfying

$$
m_{1}+2 m_{2}+\cdots+k m_{k}=k
$$

and where

$$
C\left(m_{1}, \ldots, m_{k}\right)=(-1)^{m_{1}+\cdots+m_{k}} \frac{\left(m_{1}+\cdots+m_{k}\right) !}{m_{1} ! \ldots m_{k} !} \frac{k !}{1 !^{m_{1}} \ldots k !^{m_{k}}} .
$$

For any fixed multiindex $\left(m_{1}, \ldots, m_{k}\right)$ as above, we clearly have

$$
v^{-m_{1}-\cdots-m_{k}-1}=v^{-n-1} \cdot v^{n-m_{1}-\cdots-m_{k}},
$$

the last factor on the right being bounded. Indeed,

$$
v^{n-m_{1}-\cdots-m_{k}} \in H^{\infty},
$$

since it follows from (3.3) that $n-m_{1}-\cdots-m_{k} \geq 0$. We further observe that, for $j \in \mathbf{N}$,

$$
v^{(j)}(z)=O\left((1-|z|)^{-j}\right), \quad z \in \mathbf{D}
$$

(because $v \in H^{\infty}$ ), and this implies together with (3.3) that

$$
\prod_{j=1}^{k}\left[v^{(j)}(z)\right]^{m_{j}}=O\left((1-|z|)^{-k}\right), \quad z \in \mathbf{D} .
$$

Combining (3.2) and (3.4), we see that the $k$ th summand in (3.1) takes the form $v^{-n-1} w_{k}$, where

$$
w_{k}:=\left(\begin{array}{l}
n \\
k
\end{array}\right) \sum C\left(m_{1}, \ldots, m_{k}\right) u^{(n-k)} v^{n-m_{1}-\cdots-m_{k}} \prod_{j=1}^{k}\left(v^{(j)}\right)^{m_{j}} ;
$$


the sum is understood as in (3.2). We want to show that $w_{k} \in \mathrm{BMOA}^{(n)}$, and our plan is to check the corresponding inclusion for each individual term in (3.8). Thus, we claim that the function

$$
\Phi_{m_{1}, \ldots, m_{k}}:=u^{(n-k)} v^{n-m_{1}-\cdots-m_{k}} \prod_{j=1}^{k}\left(v^{(j)}\right)^{m_{j}}
$$

satisfies

$$
\Phi_{m_{1}, \ldots, m_{k}} \in \mathrm{BMOA}^{(n)}
$$

whenever $0 \leq k \leq n$ and the $m_{j}$ 's are related by (3.3).

First let us verify (3.9) in the case $k \leq n-1$. To this end, we notice that

$$
u^{(n-k)} \in\left(H^{\infty}\right)^{(n-k)} \subset \mathrm{BMOA}^{(n-k)},
$$

where $n-k \geq 1$, while

$$
[v(z)]^{n-m_{1}-\cdots-m_{k}} \prod_{j=1}^{k}\left[v^{(j)}(z)\right]^{m_{j}}=O\left((1-|z|)^{-k}\right), \quad z \in \mathbf{D},
$$

by virtue of (3.5) and (3.7). The validity of (3.9) is then guaranteed by Lemma 2.1.

Now if $k=n$, then the multiindices involved are of the form $\left(m_{1}, \ldots, m_{n}\right)$ with $\sum_{j=1}^{n} j m_{j}=n$. For any such multiindex, at least one of the $m_{j}$ 's (say, $m_{l}$ with an $l \in\{1, \ldots, n\})$ must be nonzero, so that $m_{l} \geq 1$ and

$$
l\left(m_{l}-1\right)+\sum_{1 \leq j \leq n, j \neq l} j m_{j}=n-l .
$$

Consider the factorization

$$
\Phi_{m_{1}, \ldots, m_{n}}=v^{(l)} \cdot\left\{u v^{n-m_{1}-\cdots-m_{n}}\left(v^{(l)}\right)^{m_{l}-1} \prod_{1 \leq j \leq n, j \neq l}\left(v^{(j)}\right)^{m_{j}}\right\} .
$$

The first factor, $v^{(l)}$, is then in $\left(H^{\infty}\right)^{(l)}$ and hence in $\mathrm{BMOA}^{(l)}$, while the second factor (the one in curly brackets) is $O\left((1-|z|)^{-n+l}\right)$. The latter estimate is due to (3.6) and (3.10), coupled with the fact that $u$ and $v$ are in $H^{\infty}$. Applying Lemma 2.1 to the current factorization, we arrive at (3.9), this time with $k=n$.

Now that (3.9) is known to be true, we infer that the functions $w_{k}$ from (3.8) are all in $\mathrm{BMOA}^{(n)}$, whence obviously $\sum_{k=0}^{n} w_{k} \in \mathrm{BMOA}^{(n)}$. Recalling that

$$
f^{(n)}=v^{-n-1} \sum_{k=0}^{n} w_{k},
$$

we finally conclude that $f^{(n)}$ can be written as $g h^{(n)}$, where $g:=v^{-n-1} \in \mathcal{N}$ and $h$ is a BMOA function satisfying $h^{(n)}=\sum_{k=0}^{n} w_{k}$.

The case of $\mathcal{N}^{+}$is similar. This time, $v$ is taken to be an outer function in $H^{\infty}$, so $g=v^{-n-1}$ will be an outer function in $\mathcal{N}^{+}$.

\section{Proof of Theorem 1.3}

We shall only prove (a), the proof of (b) being similar. We know from Lemma 2.2 that $\mathcal{N} \cdot \mathrm{BMOA}^{\prime}$ is an ideal space. Furthermore, Theorem 1.1 tells us that $\mathcal{N} \cdot \mathrm{BMOA}^{\prime}$ contains $\mathcal{N}^{\prime}$. It remains to verify that, whenever $X$ is an ideal space with $\mathcal{N}^{\prime} \subset X$, 
we necessarily have

$$
\mathcal{N} \cdot \mathrm{BMOA}^{\prime} \subset X
$$

Take any $g \in \mathcal{N}$ and $h \in H^{\infty}$. Note that

$$
g h^{\prime}=(g h)^{\prime}-g^{\prime} h,
$$

where both terms on the right are in $X$. Indeed, $(g h)^{\prime}$ is obviously in $\mathcal{N}^{\prime}$ and hence in $X$, while the inclusion $g^{\prime} h \in X$ is due to the facts that $g^{\prime} \in \mathcal{N}^{\prime} \subset X$ and $h X \subset X$ (recall that $X$ is ideal). It now follows from (4.2) that $g h^{\prime} \in X$, and we have thereby checked that

$$
\mathcal{N} \cdot\left(H^{\infty}\right)^{\prime} \subset X
$$

Finally, given $\eta \in \mathrm{BMOA}$, we invoke a result of Aleksandrov and Peller [1, Theorem 3.4] to find functions $\varphi_{j}, \psi_{j} \in H^{\infty}(j=1,2)$ such that $\eta^{\prime}=\varphi_{1} \psi_{1}^{\prime}+\varphi_{2} \psi_{2}^{\prime}$. Letting $g \in \mathcal{N}$ as before, we get

$$
g \eta^{\prime}=g \varphi_{1} \psi_{1}^{\prime}+g \varphi_{2} \psi_{2}^{\prime} .
$$

Here, the two terms of the form $g \varphi_{j} \psi_{j}^{\prime}$ are in $\mathcal{N} \cdot\left(H^{\infty}\right)^{\prime}$, so we infer from (4.3) that they are also in $X$. The right-hand side of (4.4) is therefore in $X$, and so is the left-hand side, $g \eta^{\prime}$. Thus we conclude that $g \eta^{\prime} \in X$ for all $g \in \mathcal{N}$ and $\eta \in$ BMOA. This establishes (4.1) and completes the proof.

\section{References}

[1] Aleksandrov, A. B., and V. V. Peller: Hankel operators and similarity to a contraction. - Internat. Math. Res. Notices 6, 1996, 263-275.

[2] Cohn, W.S.: A factorization theorem for the derivative of a function in $H^{p}$. - Proc. Amer. Math. Soc. 127, 1999, 509-517.

[3] Cohn, W. S., and I. E. Verbitsky: Factorization of tent spaces and Hankel operators. - J. Funct. Anal. 175, 2000, 308-329.

[4] Dyakonov, K. M.: Zero sets and multiplier theorems for star-invariant subspaces. - J. Anal. Math. 86, 2002, 247-269.

[5] Garnett, J. B.: Bounded analytic functions. - Springer, New York, revised first edition, 2007.

[6] Hayman, W. K.: On the characteristic of functions meromorphic in the unit disk and of their integrals. - Acta Math. 112, 1964, 181-214.

[7] Horowitz, C.: Zeros of functions in the Bergman spaces. - Duke Math. J. 41, 1974, 693-710.

[8] Jevtić, M.: On the Carleson measure characterization of BMOA functions on the unit ball. - Proc. Amer. Math. Soc. 114, 1992, 379-386.

[9] Schwartz, L.: Cours d'analyse. - Hermann, Paris, second edition, 1981.

[10] Shamoyan, F. A.: Toeplitz operators in some spaces of holomorphic functions and a new characterization of the class BMO. - Izv. Akad. Nauk Armyan. SSR Ser. Mat. 22:2, 1987, $122-132$.

[11] Triebel, H.: Theory of function spaces. II. - Monographs in Mathematics 84, Birkhäuser Verlag, Basel, 1992.

[12] Yanagihara, N.: On a class of functions and their integrals. - Proc. London Math. Soc. (3) $25,1972,550-576$. 